\title{
Video Recording of Nursing Care Activities in Gerontological Nursing to Compare General Units and Comprehensive Nursing Care Units
}

\author{
Bae, Sung-Heui ${ }^{1} \cdot$ Kim, Jeonghyun ${ }^{2} \cdot$ Lee, Inyoung ${ }^{2} \cdot$ Oh, Seung Jin $^{3} \cdot$ Shin, Sujin ${ }^{1}$ \\ ${ }^{1}$ Associate Professor, College of Nursing, Ewha Womans University, Seoul \\ ${ }^{2}$ Doctoral Student, College of Nursing, Ewha Womans University, Seoul \\ ${ }^{3}$ Team Leader, Department of International Cooperation, Korean Nurses Association, Seoul, Korea
}

\begin{abstract}
Purpose: Comprehensive nursing care services are provided for patient-centered nursing care in Korea. This study was conducted to compare differences in the frequency and elapsed time of nursing activities between comprehensive nursing care units and general medical-surgical units. Methods: This is a descriptive study design using direct observation of nursing care activities. A time and motion study on the frequency and elapsed time of nursing activities provided by nurses was conducted in the comprehensive nursing care units and general units of two small-to-medium sized hospitals. Data were collected for 64 hours in each hospital, through a two-way method using a checklist and a camera recorder. Results: The total number of nursing activities, activities provided per patient and patient safety care including clinical assessment and treatment were higher in the comprehensive nursing care units compared to general units. We found similar findings regarding the duration of nursing activities. Conclusion: Comprehensive nursing care units provide patient-centered nursing care and have a broader range of nursing activities for patient safety than general units. Assessments conducted to meet nursing needs and to promote safe nurse staffing should reflect the characteristics of the nursing tasks performed in comprehensive nursing care units.
\end{abstract}

Key Words: Nursing care; Workforce; Patient-centered care; Patient safety; Video recording

\section{INTRODUCTION}

\section{Background}

There are great concerns about safe nurse staffing to provide appropriate healthcare services worldwide [1]. In Korean culture, inpatient care has traditionally been considered as a responsibility of the family [2]. To resolve this problem, the government implemented a pilot trial of comprehensive nursing care services in 2013 to reduce the care burden of families and to improve patient safety. A team nursing system was adopted, which was composed of professional healthcare personnel who provide holistic nursing services, including care previously provided by family members. After the outbreak of the Middle East respiratory syndrome in 2015, which revealed system failure of the existing hospitalization system that threatened patient safety, the government established a framework for patient-centered nursing care by accelerating the expansion of the number of comprehensive nursing care units (CNUs) and by improving inpatient-reimbursement systems and related health policy [3].

The comprehensive nursing care service is intended to provide holistic nursing services to patients by nurses in hospitals; thus, the nurse to patient ratio was arranged to be 1:5 7 for tertiary hospitals, and 1:10 16 for primary hospitals. This nurse-to-patient ratios are higher than current nurse to patient ratio in general units [4]. Consequently, implementation of the comprehensive nursing care service resulted in a major change in the scope of nursing care, as nursing activities previously provided by patients' family members or informal caregivers became the responsibility of nurses and other nursing staff [5].

Various aspects of general nursing care not directly

Corresponding author: Shin, Sujin https://orcid.org/0000-0001-7981-2893

College of Nursing, Ewha Womans University, 52 Ewhayeodae-gil, Seodaemun-gu, Seoul 03760, Korea.

Tel: +82-2-3277-2726, Fax: +82-2-3277-2850, E-mail: ssj1119@ewha.ac.kr

Received: Aug 14, 2019 / Revised: Sep 25, 2019 / Accepted: Oct 16, 2019

This is an open access article distributed under the terms of the Creative Commons Attribution Non-Commercial License (http://creativecommons.org/licenses/ by-nc/3.0), which permits unrestricted non-commercial use, distribution, and reproduction in any medium, provided the original work is properly cited. 
provided by nurses in the general units (GUs), such as oral care, bathing, and meal assistance, were included in the nursing activities in the CNUs [6]. Additionally, there is greater emphasis on managing patient well-being through measures such as pain assessments with regular observation of patients, and communication with patients such as patient education, counseling, emotional support and the duration of contact was increased $[7,8]$. Previous studies found that the increase in the proportion of direct nursing care provided to inpatients in CNUs resulted in positive outcomes, such as decrease in patient safety incidents; improvements in patient indicators including pressure ulcers and falls; and increases in patients' satisfaction with nursing care service $[9,10]$.

At a time when the elderly population is growing rapidly and the importance of managing chronic illness emerges, the need for CNUs is increasing because such positive outcomes of $\mathrm{CNUs}$ are necessary for nursing care of the older population [11]. However, the service brought new problems that had not been encountered in the GUs, including work overload exceeding the nurse staffing level, ambiguity of nursing activities among the nursing staff, and unnecessary personal requests from patients [5]. Therefore, the differences in the nursing activities between CNUs and GUs have received attention. A comprehensive investigation of differences in the nursing activities between CNUs and GUs, is required for stable implementation of the comprehensive nursing care services system to systematically analyze nursing needs and safe nurse staffing. However, previous studies have only analyzed the frequency of nursing activities by using electronic nursing record [6] or self-administered surveys, which have limitations, as these studies have failed to analyze the time spent to provide various nursing activities [7]. The only observational study that has been conducted presented the level of nurse staffing required for CNUs, based on an analysis of nursing activities and average daily working hours of nurses in GUs in a tertiary hospital [12].

Therefore, this study was done to closely examine the nursing activities in GUs and in CNUs using a two-way method including direct observation and camera recording in small-medium sized hospitals. Nursing activities were compared according to the characteristics of each unit and by shift. By investigating the nursing activities through direct observation, this study hoped to provide important knowledge to support and staffing management and improvements of patient safety in CNUs in the future.

\section{Aim of Study}

This study was conducted to compare nursing activities between CNUs and GUs in small-to-medium sized hospitals, and the specific objectives of the study were as follows.

- To compare the frequency and elapsed time of each nursing activity provided by nurses working in CNUs and GUs.

- To compare the frequency and elapsed time of nursing activities provided for each patient in CNUs and GUs.

\section{METHODS}

\section{Study Design}

This was a descriptive study conducted to analyze differences in nursing activities between CNUs and GUs.

\section{Sample and Settings}

An observational study was conducted in two smallto-medium sized hospitals located in Seoul during June and July 2018. We examined the frequency and elapsed time of direct and indirect nursing activities provided by nurses during a 64-hour period in CNUs and in GUs during the day and evening shifts. The observed CNUs utilized team nursing care delivery models for $\mathrm{CNUs}$ and as the functional nursing care delivery models commonly used in small-to-medium hospitals for GUs. The nurses to be observed were selected as nurses with at least three months of experience in CNUs or in GUs.

\section{Data Collection}

A time and motion study was conducted to measure the frequency and elapsed time of nursing activities. Data were collected only for the day and evening shifts during the 4 days considering the characteristics of small-to-medium sized hospitals, which minimize nursing activities and staff during the night shift. To precisely examine the direct and indirect nursing activities and the elapsed time of those activities, data were collected using a two-way method in which the frequency of nursing activities was recorded on a checklist developed for this observational study and the nursing activities were recorded with a video camera.

A total of 4 investigators were involved in this study, all of whom received relevant training prior to data 
collection. The training section includes the purpose of study, items in the checklist, how to use the camcorder, and how to measure the elapsed time. Accompanied by a nurse who agreed to participate in the study, an investigator videotaped nursing activities on each shift during the study period, and simultaneously recorded using the checklist. Nursing activities were measured from the start of the night-day shift handover until the end of the evening-night shift handover. The starting point of each nursing activity was the time of arrival in the patient room, and the ending point was the time that the nurse left the patient room after providing the nursing care for every single activity, and the time before the start of the next nursing activity for multiple activity. Medication and peripheral intravenous (IV) line treatment were divided into medication and IV preparation as an indirect nursing activity and medication and IV-line administration as a direct nursing activity. The camcorder used for making video recordings was a JW-8600 model (JUN WORLD, Seoul, Korea). The nursing activities were recorded in an Audio Video Interleave (AVI) file format with resolution of $1920 \times 1080$. The GUs utilized a functional nursing care delivery model, whereas the CNUs utilized a team nursing delivery model; thus, data collection and analysis were conducted by following the "acting nurse" who is responsible for all of the nursing activities within the GU.

The items on the checklist were adopted from the Korean Patient Classification System (KPCS-1) developed by Song et al.[13] the items of direct nursing activities presented in the study by Moon et al. [14], and the items of indirect nursing activities presented in the studies by Moon et al.[14] and by Hwang [15] and modified by the authors.

\section{Data Analysis}

The frequency and the elapsed time of each nursing activity were analyzed in Microsoft Excel 2016 based on the data collected by the checklist. The frequency and the elapsed time of the nursing activities provided per patient were obtained as mean values by dividing the corresponding values in data collected from each work shift by the number of patients overseen by the nurse during the corresponding shift. Seventy-one gigabytes of data were collected using the camcorder; these videos were utilized to increase the overall reliability of data through a precise examination of whether the items were appropriately marked on the checklist during the analysis, as well as to evaluate the elapsed time of nursing activities.

\section{Ethical Considerations}

This study received approval from the Institutional Review Board of *** University (No. 153-13). Data was collected from nurses after obtaining informed consent from those who agreed to participate in the study. During data collection, the nurses and the patients were informed about the process of data collection for research. Persons and activities other than the nursing activities of the nurses who agreed to participate in the study were not recorded, and video recordings were discontinued when a patient's face or other body parts were exposed.

\section{RESULTS}

\section{General Characteristics of the Investigated units}

Table 1 presents the general characteristics of the 2 CNUs and the 2 GUs of the 2 hospitals that participated in this observational study. Nurses working in the CNUs, which utilized a team nursing delivery model, were each responsible for 10 13 patients; however, nurses working in the GUs, which utilize a functional nursing care delivery model, were each responsible for $25 \sim 28$ patients, and only 1 or 2 nurses were responsible for the nursing activities of the entire unit (Table 1).

Table 1. General Characteristics of the Investigated Units

\begin{tabular}{|c|c|c|c|c|}
\hline \multirow{2}{*}{ Variables } & \multicolumn{2}{|c|}{$\mathrm{CNU}$} & \multicolumn{2}{|c|}{ GU } \\
\hline & A1 & A2 & B1 & B2 \\
\hline Number of beds & 41 & 45 & 31 & 54 \\
\hline Number of nurses & 17 & 20 & 9 & 12 \\
\hline Nursing care delivery model & Team nursing & Team nursing & Functional nursing & Functional nursing \\
\hline Number of patient per nurse & $10 \sim 13$ & $10 \sim 12$ & $26 \sim 28^{*}$ & $25 \sim 27^{*}$ \\
\hline
\end{tabular}

*The number of patient of "acting nurse" who is responsible for all nursing activities within the ward; CNU=Comprehensive nursing units; GU=General units. 


\section{Nursing Activities Provided by Nurses}

\section{1) Frequency and elapsed time of each nursing activity}

The frequency of direct nursing services performed in the CNUs was as follows: $36.9 \%$ time for medication and intravenous (IV) line administration, followed by $18.8 \%$ for assessment and monitoring. In the GUs, there were $42.9 \%$ of medication and IV-line administration, which also accounted for the highest percentage. A difference from the CNUs, however, was that measurements were the next most frequently-performed activity (19.1\%). Assessment and monitoring exhibited higher frequency in the CNUs than in the GUs. The activity with the greatest difference in frequency was assessment and monitoring, which accounted for $18.8 \%$ of time in the CNUs and $7.7 \%$ in the GUs (Table 2).

Upon examination of indirect nursing activities, information management accounted for the highest percentage of time spent in both the CNUs and GUs, accounting for $38.0 \%$ and $30.7 \%$, respectively.

Among the direct nursing activities, medication and IV-line administration took 407 minutes (39.5\%) in the CNUs and 527 minutes (44.0\%) in the GUs; in both types of wards, medication and IV-line administration required the greatest amount of time among the total direct nursing activities. However, there were differences between the two wards in the subsequent order of the time required by other direct nursing activities. In the CNUs, medication and IV line administration was followed by assessment and monitoring (148 minutes, 14.4\%) and then by nursing interventions (140 minutes, $13.6 \%$ ), whereas it was followed by measurements (167 minutes, $14.0 \%)$ and patient education (147 minutes, $12.3 \%$ ) in the GUs. More time was taken by assessment and monitoring, nursing interventions, nutrition/elimination, and respiratory care in the CNUs than in the GUs, and the direct nursing activity with the greatest difference in the percentage of elapsed time was assessment and monitoring, which accounted for 148 minutes $(14.4 \%)$ in the CNUs, but only 74 minutes (6.2\%) in the GUs. Additionally, the percentages of elapsed time of medication and IV-line administration $(44.0 \%)$ and patient education $(12.3 \%)$ were greater in the GUs than in the CNUs (39.5\% and 6.3\%, respectively).

Although information management and communication accounted for the greatest amount of time among the indirect nursing activities in both wards, information management accounted for more time in the CNUs (674 minutes, $42.6 \%$ ) than in the GUs (597 minutes, 37.6\%).

\section{2) Frequency and elapsed time of nursing activities provided per patient}

The frequency and the elapsed time of nursing activities provided per patient on each shift were 9.55 events and 30.42 minutes, respectively, in the CNUs, and 4.40 events and 12.89 minutes, respectively, in the GUs; thus, patients in the CNUs had longer, more frequent contact with the nurses than patients in the GUs (Table 3).

The frequency of all direct nursing activities and the

Table 2. Nursing Activities Provided by Nurses

\begin{tabular}{|c|c|c|c|c|c|}
\hline \multirow{3}{*}{ Variables } & \multirow{3}{*}{ Categories } & \multicolumn{4}{|c|}{ Frequency and elapsed time } \\
\hline & & \multicolumn{2}{|c|}{$\mathrm{CNU}$} & \multicolumn{2}{|c|}{ GU } \\
\hline & & $\mathrm{n}(\%)$ & $\operatorname{Min}(\%)$ & $\mathrm{n}(\%)$ & $\operatorname{Min}(\%)$ \\
\hline \multirow{11}{*}{$\begin{array}{l}\text { Direct } \\
\text { nursing }\end{array}$} & Total & $517(100.0)$ & $1,031(100.0)$ & $601(100.0)$ & $1,197(100.0)$ \\
\hline & Medication and IV-line administration & $191(36.9)$ & 407 (39.5) & $258(42.9)$ & $527(44.0)$ \\
\hline & Assessment and monitoring & $97(18.8)$ & $148(14.4)$ & $46(7.7)$ & $74(6.2)$ \\
\hline & Measurement & $72(13.9)$ & $138(13.4)$ & $115(19.1)$ & $167(14.0)$ \\
\hline & Patient education & $40(7.7)$ & $65(6.3)$ & $66(11.0)$ & $147(12.3)$ \\
\hline & Nursing intervention & $34(6.6)$ & $140(13.6)$ & $19(3.2)$ & $108(9.0)$ \\
\hline & Safety management & $32(6.2)$ & $37(3.6)$ & $57(9.5)$ & $62(5.2)$ \\
\hline & Mobilization and positioning & $24(4.6)$ & $35(3.4)$ & $23(3.8)$ & $69(5.8)$ \\
\hline & Respiratory care & $11(2.1)$ & $21(2.0)$ & $3(0.5)$ & $8(0.6)$ \\
\hline & Hygiene & $9(1.8)$ & $10(1.0)$ & $13(2.2)$ & $31(2.6)$ \\
\hline & Nutrition/elimination & $7(1.4)$ & $30(2.8)$ & $1(0.1)$ & $4(0.3)$ \\
\hline \multirow{6}{*}{$\begin{array}{l}\text { Indirect } \\
\text { nursing }\end{array}$} & Total & $305(100.0)$ & $1,584(100.0)$ & $348(100.0)$ & $1,588(100.0)$ \\
\hline & Information management & $116(38.0)$ & $674(42.6)$ & $107(30.7)$ & $597(37.6)$ \\
\hline & Medication and IV preparation & $86(28.2)$ & $181(11.4)$ & $102(29.3)$ & $257(16.2)$ \\
\hline & Communication & $70(23.0)$ & $671(42.4)$ & $76(21.8)$ & $588(37.0)$ \\
\hline & Environmental control & $32(10.5)$ & $56(3.5)$ & $56(16.1)$ & $124(7.8)$ \\
\hline & Drug control & $1(0.3)$ & $2(0.1)$ & $7(2.1)$ & $22(1.4)$ \\
\hline
\end{tabular}

$\mathrm{IV}=$ Intravenous; $\mathrm{CNU}=$ Comprehensive nursing units; $\mathrm{GU}=\mathrm{General}$ units. 
Table 3. Frequency and Elapsed Time of Nursing Activities Provided per Patient

\begin{tabular}{|c|c|c|c|c|c|}
\hline \multirow{3}{*}{ Variables } & \multirow{3}{*}{ Categories } & \multicolumn{2}{|c|}{$\begin{array}{c}\text { Frequency of nursing activities } \\
\text { provided per patient }\end{array}$} & \multicolumn{2}{|c|}{$\begin{array}{c}\text { Elapsed time of nursing activities } \\
\text { provided per patient }\end{array}$} \\
\hline & & $\mathrm{CNU}$ & GU & $\mathrm{CNU}$ & GU \\
\hline & & $\mathrm{n}(\%)$ & $\mathrm{n}(\%)$ & $\operatorname{Min}(\%)$ & $\operatorname{Min}(\%)$ \\
\hline Total & & $9.55(100.0)$ & $4.40(100.0)$ & $30.42(100.0)$ & $12.89(100.0)$ \\
\hline $\begin{array}{l}\text { Direct } \\
\text { nursing }\end{array}$ & $\begin{array}{l}\text { Subtotal } \\
\text { Medication and IV-line administration } \\
\text { Assessment and monitoring } \\
\text { Measurement } \\
\text { Patient education } \\
\text { Nursing intervention } \\
\text { Safety management } \\
\text { Mobilization and positioning } \\
\text { Respiratory care } \\
\text { Hygiene } \\
\text { Nutrition/elimination }\end{array}$ & $\begin{array}{l}6.04(100.0) \\
2.25(37.2) \\
1.13(18.8) \\
0.84(13.8) \\
0.46(7.7) \\
0.38(6.3) \\
0.38(6.2) \\
0.29(4.7) \\
0.12(2.0) \\
0.11(1.8) \\
0.08(1.5)\end{array}$ & $\begin{array}{l}2.79(100.0) \\
1.20(43.0) \\
0.21(7.7) \\
0.54(19.2) \\
0.30(10.8) \\
0.09(3.1) \\
0.27(9.5) \\
0.11(3.8) \\
0.01(0.5) \\
0.05(2.2) \\
0.01(0.2)\end{array}$ & $\begin{array}{l}11.97(100.0) \\
4.75(39.7) \\
1.70(14.2) \\
1.62(13.5) \\
0.75(6.2) \\
1.60(13.3) \\
0.44(3.7) \\
0.41(3.5) \\
0.23(1.9) \\
0.11(1.0) \\
0.36(3.0)\end{array}$ & $\begin{array}{l}5.56(100.0) \\
2.46(44.2) \\
0.35(6.2) \\
0.78(13.9) \\
0.67(12.1) \\
0.50(9.0) \\
0.29(5.2) \\
0.31(5.6) \\
0.03(0.7) \\
0.15(2.7) \\
0.02(0.4)\end{array}$ \\
\hline $\begin{array}{l}\text { Indirect } \\
\text { nursing }\end{array}$ & $\begin{array}{l}\text { Subtotal } \\
\text { Information management } \\
\text { Medication and IV preparation } \\
\text { Communication } \\
\text { Environmental control } \\
\text { Drug control }\end{array}$ & $\begin{array}{l}3.51(100.0) \\
1.33(37.9) \\
1.00(28.4) \\
0.79(22.4) \\
0.38(11.0) \\
0.01(0.3)\end{array}$ & $\begin{array}{l}1.61(100.0) \\
0.49(30.6) \\
0.47(29.5) \\
0.35(21.7) \\
0.26(16.2) \\
0.04(2.0)\end{array}$ & $\begin{array}{l}18.45(100.0) \\
7.84(42.5) \\
2.10(11.4) \\
7.81(42.3) \\
0.68(3.7) \\
0.02(0.1)\end{array}$ & $\begin{array}{l}7.33(100.0) \\
2.75(37.5) \\
1.20(16.3) \\
2.71(36.9) \\
0.57(7.8) \\
0.10(1.5)\end{array}$ \\
\hline
\end{tabular}

$\mathrm{IV}=$ Intravenous; $\mathrm{CNU}=$ Comprehensive nursing units; $\mathrm{GU}=$ General units.

elapsed time of all activities, excluding hygiene, were greater in the CNUs. Medication and IV-line administration was the most frequent direct nursing activity in both the CNUs (37.2\%) and the GUs (43.0\%). The next most frequent direct nursing activity in the CNUs was assessment and monitoring $(18.8 \%)$, followed by measurements (13.8 $\%)$, and the next most frequent activity in the GUs was measurements $(19.2 \%)$, followed by patient education $(10.8 \%)$. The elapsed time of direct nursing activities in the order of longest to shortest in the CNUs was medication and IV-line administration (4.75 minutes, 39.7\%), assessment and monitoring (1.70 minutes, $14.2 \%)$, and measurements (1.62 minutes, $13.5 \%)$. The corresponding order in the GUs was medication and IV-line administration ( 2.46 minutes, $44.2 \%$ ), measurements ( 0.78 minutes, $13.9 \%)$, and patient education ( 0.67 minutes, $12.1 \%)$.

\section{DISCUSSION}

This study was conducted to examine the differences in nursing activities provided per patient between CNUs and GUs, and can provide important knowledge regarding nursing activities and nursing needs in CNUs.

Although the frequency and elapsed time of all direct nursing activities were higher in the GUs than in the $\mathrm{CNUs}$, the frequency and elapsed time of direct nursing activities provided per patient were higher in the CNUs. This discrepancy may be attributed to the difference in the number of patients per nurse. The CNU utilizes a team nursing model, and thus the subjects of the study were the nurses responsible for a single team (about 10 13 patients). In contrast, the general unit utilizes a functional nursing care delivery model, and the subjects were therefore the acting nurses responsible for the nursing activities of the entire unit (about 25 28 patients).

The elapsed time of direct nursing activities provided per patient in the CNUs (based on an 8-hour shift) was 11.97 minutes, which was more than twice that of the GUs (5.56 minutes). These results indicate that patients in the CNUs, with less number of patients per nurse, had more frequent and longer interact with nurses than patients in the GUs. In a previous study, the average number of patients per nurse in CNUs of secondary hospitals was 10 [16], similar to the result of our study. This ratio is slightly different from that of GUs of secondary hospitals, in which one nurse was responsible for approximately 18 patients. Additionally, the elapsed time of direct nursing activities in CNUs presented in this study was similar to previous findings (11.3 minutes) among nurses in GUs of tertiary hospitals and secondary hospitals with more than 400 beds [12]. The finding that the level of nurse staffing of CNUs is similar to that of GUs in tertiary hospitals 
(9.6 12.0 patients per nurse) indicates that nurses working in CNUs can provide a similar amount of per-patient nursing time to that of tertiary hospitals, which have patients with greater disease severity.

Appropriate level of nurse staffing is important for patient safe, high-quality nursing care. The American Nurses Association has emphasized that 'Safe Staffing Saves Lives'-that is, that appropriate levels of nurse staffing is needed for patient safety. Reducing the number of patients per nurse has positive outcomes, including decreases in patients' readmission and mortality rates $[17,18]$, decreases in the number of falls, pressure ulcers, and nosocomial infections [19-21], and prevention of nurse exhaustion and job dissatisfaction [22]. Because of these positive outcomes, mandated minimum nurse staffing ratios have been applied in California, USA, limiting the nurse-to-patient ratio of the general medical-surgical unit to 1:5[23]. In Japan, the nurse staffing indicator has been changed to include the number of nurses who provide actual nursing care per patient rather than the total number of nurses employed per patient, and a new category of general care unit was introduced with a higher nurse staffing standard of a 1:7 nurse-to-patient ratio [24]. Therefore, the expansion of $\mathrm{CNUs}$ suggests that the number of patients per nurse can be reduced, which can be a health policy to improve recruitment and retention of nursing staff to promote patient safety and quality improvements in nursing care.

In terms of specific nursing activities, the frequency and the proportion of elapsed time of the activities such as assessment and monitoring were more than 2 times higher in the CNUs than in the GUs. This implies that assessments of patients' conditions were more effectively performed in the CNUs than in the GUs. This might be difficult in prioritizing assessment and monitoring due to shortage of nursing staff in GUs. These results are consistent with the findings of previous studies reporting that CNUs showed high performance levels of assessment and monitoring activities [25] and a high frequency of nursing activities associated with patient assessment, such as nurse rounding and pain assessment [6]. Furthermore, communication, including nursing handovers, accounted for a high percentage of elapsed time of indirect nursing activities in the CNUs. This also demonstrates that the CNUs provided more patient-centered nursing care based on a thorough, complete assessment of the patient's condition and patient-nurse communication than the GUs. These advantages are important for providing nursing care to elderly patients. Elderly patients have high co-morbidities with several chronic diseases, and the compensatory capacity of body is reduced, so the rate of disease progression and the manifestation pattern are often different [26]. In addition, more close assessment and patient-nurse communication are needed in patients affected by senility-associated diseases such as stroke and dementia [27]. Therefore, in comparison with GUs, CNUs may be a nursing practice environment that provides both nursing care and emotional support that correspond to the needs of the patients including the elderly through a holistic, therapeutic relationship.

Despite the low nurse to patient ratio in CNUs, outcomes such as job satisfaction, role ambiguity, and work stress varied among studies [5,6]. According to previous studies $[7,28]$, CNUs brought forth new problems that had not been encountered in GUs, including work overload exceeding the standardized nurse staffing [28]. These problems suggest a need to review the current method of nurse staffing, which does not consider the characteristics of the nursing care performed in CNUs or the work intensity of each shift. In Japan, specialized nurses who assess nursing needs and the patient severity continuously evaluate in the nursing unit, and promptly respond to situations that require greater nursing needs by adjusting the nurse staffing. For example, they might designate a higher number of nurses in a week with greater nursing needs due to factors such as patient fluctuation or patient severity $[29,30]$. Strategies for efficient management such as variations in nurse staffing must also be investigated in Korea in order to reduce job stress and demand among nurse staff and to improve both the inpatient satisfaction and patient safety.

This study does not reflect the diagnosis and severity of patients in the analysis of nursing needs and does not include the complexity of nursing activities in the classification and comparison of nursing activities. Furthermore, if the observed nurses' career experience had been controlled to a similar level, more valid results could have been obtained. Despite these limitations, this study is significant in that it presents an analysis of data acquired via a direct observational study of approximately 1,770 nursing activities over a total of 128 hours. In addition, a meaningful aspect of this study is that the reliability of the data was ensured by using a two-way method of data collection that combined the use of a checklist with video recordings.

\section{CONCLUSION}

In this study an analysis was done of the differences in the frequency and elapsed time of nursing activities, as 
well as differences in the nursing activities provided per patient, between CNUs and GUs. The results of this study show that direct nursing activities overall, as well as the frequency and elapsed time of nursing activities provided per patient, were higher in the CNUs, indicating that they adequately provided safe, patient-centered nursing care. The characteristics and benefits of CNUs observed in this study, which are a high staffing level and support for patient - centered nursing care on comprehensive nursing service wards analyzed through the results of this study suggest directions for future improvements in the nursing environment.

Based on the results, we present the following suggestions. First, an observational study should be conducted with a comparative analysis of differences in nursing activities among additional types of medical institutions, such as tertiary hospitals or primary hospitals. Second, appropriate staff training and improvements in the evaluation of nursing needs in a way that accounts for differences in nursing activities between CNUs and GUs are required.

\section{CONFLICTS OF INTEREST}

The authors declared no conflict of interest.

\section{AUTHORSHIP}

Study conception and design acquisition - BSH, OSJ and SS; Data collection - KJ and OSJ; Analysis and interpretation of the data $\mathrm{KJ}$ and LI; Critical revision of the manuscript - BSH and SS.

\section{ACKNOWLEDGEMENT}

This work was supported by the Ministry of Education of the Republic of Korea and the National Research Foundation of Korea (NRF-2017S1A5A2A03067637).

\section{REFERENCES}

1. Aiken LH, Fagin CM. Evidence-based nurse staffing: ICN's new position statement. International Nursing Review. 2018; 65(4):469-71. https://doi.org/10.1111/inr.12499

2. Park DJ. The introduction of comprehensive nursing services and care problems in Korea. Monthly Welfare Trend. 2015; 202(1):21-4.

3. Ministry of Health and Welfare. Comprehensive nursing services participation status [Internet]. Sejong: Ministry of Health and Welfare; 2019 [updated 2019 Aug 14; cited 2019 Oct 7]. Available from: http://www.mohw.go.kr/react/gm/sgm0601vw.jsp?PAR_ MENU_ID=13\&MENU_ID=1304020401\&page=1\&CONT_S $\mathrm{EQ}=327656$
4. National Health Insurance Service. Comprehensive nursing care service standard operation guideline. Wonju: National Health Insurance Service; 2018. 15 p.

5. Kim SE, Han JY. Clinical nurses' job stress, emotional labor, nursing performance, and burnout in comprehensive nursing care service wards and general wards. Journal of Korean Academy Nursing Administration. 2017;23(3):336-45.

https://doi.org/10.11111/jkana.2017.23.3.336

6. LeeSS, Yu S. Comparison analysis between integrated nursing care service and non-integrated nursing care service through electronic nursing documentation. Journal of Health Informatics and Statistics. 2017;42(2):170-6.

https://doi.org/10.21032/jhis.2017.42.2.170

7. Ahn SH, Jung SH, You JH, Lee MA. Nursing task and practice environment for nursing work perceived by nurses working on comprehensive wards versus general wards. Journal of Korean Academy Nursing Administration. 2018;24(1):10-20. https://doi.org/10.11111/jkana.2018.24.1.10

8. Park KO, Yu M, Kim JK. Experience of nurses participating in comprehensive nursing care. Journal of Korean Academy of Nursing Administration. 2017;23(1):76-89. https://doi.org/10.11111/jkana.2017.23.1.76

9. Jung YA, Sung KM. A comparison of patients' nursing service satisfaction, hospital commitment and revisit intention between general care unit and comprehensive nursing care unit. Journal of Korean Academy Nursing Administration. 2018;24 (1):30-9. https:// doi.org/10.11111/jkana.2018.24.1.30

10. Park JH, Lee MH. Effects of nursing and care-giving integrated service on nursing work performance, nurses' job satisfaction and patient safety. Journal of Korean Academy Social Home Care Nursing. 2017;24(1):14-22. https://doi.org/10.22705/jkashcn.2017.24.1.014

11. Roh HJ, Kim EJ, Sok SH. Influences of nurses' empathy and self-efficacy on nursing care of older adults in an integrated nursing care services (INCS) unit. Journal of East-West Nursing Research. 2019;25(1):9-16. https://doi.org/10.14370/jewnr.2019.25.1.9

12. Cho SH, Song KJ, Park IS, Kim YH, Kim MS, Gong DH, et al. Development of staffing levels for nursing personnel to provide inpatients with integrated nursing care. Journal of Korean Academy Nursing Administration. 2017;23(2):211-22. https://doi.org/10.11111/jkana.2017.23.2.211

13. Song KJ, Kim EH, Yoo CS, Park HO, Park KO. Development of KPCS (Korean Patient Classification System for Nurses) based on nursing needs. Journal of Korean Clinical Nursing Research. 2009;15(1):5-17.

14. Moon SN, Kim YJ, Park JH, Choi JA. A fundamental study to measure the nursing workload in military hospitals-grouping a list of direct/indirect nursing activities and producing an average nursing minutes by processing a nursing activities 
time. Journal of Military Nursing Research. 2007;25(1):63-98.

15. Hwang JI. Impact of computerization on direct and indirect nursing time. Journal of East-West Nursing Research. 2005;11 (1):58-64.

16. Yoon HS, Lim JY, Kang MJ. Comparison of expectation-perception between patient and nurse on nursing care service in comprehensive nursing care wards. Journal of the Korea Contents Association. 2017;17(3):507-22. https://doi.org/10.5392/JKCA.2017.17.03.507

17. Lasater KB, Mchugh MD. Nurse staffing and the work environment linked to readmissions among older adults following elective total hip and knee replacement. International Journal for Quality in Health Care. 2016;28(2):253-8. https://doi.org/10.1093/intqhc/mzw007

18. Everhart D, Schumacher JR, Duncan RP, Hall AG, Neff DF, Shorr RI. Determinants of hospital fall rate trajectory groups: a longitudinal assessment of nurse staffing and organizational characteristics. Health Care Management Review. 2014; 39(4):352-60. https://doi.org/10.1097/HMR.0000000000000013

19. Choi J, Staggs VS. Comparability of nurse staffing measures in examining the relationship between RN staffing and unit-acquired pressure ulcers: a unit-level descriptive, correlational study. International Journal of Nursing Studies. 2014;51(10): 1344-52. https://doi.org/10.1016/j.ijnurstu.2014.02.011

20. Hirsbrunner T, Denhaerynck K, Fierz K, Milisen K, Schwendimann R. Nurse staffing, patient turnover and safety climate and their association with in-patient falls and injurious falls on medical acute care units: a cross-sectional study. Journal of Hospital Administration. 2015;4(3):54-60. https://doi.org/10.5430/jha.v4n3p54

21. Shang J, Stone P, Larson E. Studies on nurse staffing and health care-associated infection: methodologic challenges and potential solutions. American Journal of Infection Control. 2015;43(6):581-8.

https://doi.org/10.1016/j.ajic.2015.03.029
22. Shin SJ, Park JH, Bae SH. Nurse staffing and nurse outcomes: a systematic review and meta-analysis. Nursing Outlook. 2018;66(3):273-82. https://doi.org/10.1016/j.outlook.2017.12.002

23. Olley R, Edwards I, Avery M, Cooper H. Systematic review of the evidence related to mandated nurse staffing ratios in acute hospitals. Australian Health Review. 2018;43(3):288-93. https://doi.org/10.1071/AH16252

24. Morioka N, Tomio J, Seto T, Kobayashi Y. The association between higher nurse staffing standards in the fee schedules and the geographic distribution of hospital nurses: a cross-sectional study using nationwide administrative data. BMC Nursing. 2017;16(1):25.

https://doi.org/10.1186/s12912-017-0219-1

25. Lee MK, Jung DY. A study of nursing tasks, nurses' job stress and job satisfaction in hospitals with no guardians. Journal of Korean Academy Nursing Administration. 2015;21(3):287-96. https://doi.org/10.11111/jkana.2015.21.3.287

26. Kim CO. Co-occurrence of chronic diseases and geriatric syndromes. Korean Journal of Clinical Geriatrics. 2018;19(1):23-6. https://doi.org/10.15656/kjcg.2018.19.1.23

27. Mun JY, Sohn EN. Topic manipulation skill of the elderly while aging. Journal of Speech-Language \& Hearing Disorders. 2019; 28(1):95-101. https://doi.org/10.15724/jslhd.2019.28.1.095

28. Kim JH, Kim SJ, Park ET, Jeong SY, Lee EH. Policy issues and new direction for comprehensive nursing service in the National Health Insurance. Journal of Korean Academy Nursing Administration. 2017;23(3):312-22.

https://doi.org/10.11111/jkana.2017.23.3.312

29. You SJ. Policy implications of nurse staffing legislation. Journal of the Korea Contents Association. 2013;13(6):380-9. https://doi.org/10.5392/JKCA.2013.13.06.380

30. Park BK, Bu EH, Park YS, Jang JB, Kim MR, Ahn GH, et al. Comprehensive nursing care service improvement plan 2017. Gyeonggi: National Health Insurance Service Ilsan Hospital; 2017. Report No. 2017-20-001 